\title{
Opto-thermal simulation model for optimizing laser-excited remote phosphor systems
}

Elisavet Chatzizyrli, Nadine Tinne, Roland Lachmayer, Jörg Neumann, Dietmar Kracht

Elisavet Chatzizyrli, Nadine Tinne, Roland Lachmayer, Jörg Neumann, Dietmar Kracht, "Opto-thermal simulation model for optimizing laser-excited remote phosphor systems," Proc. SPIE 10693, Illumination Optics V, 1069300 (28 May 2018); doi: 10.1117/12.2312644 


\title{
Opto-thermal simulation model for optimizing laser-excited remote phosphor systems
}

\author{
Elisavet Chatzizyrli**, ${ }^{\mathrm{a}, \mathrm{b}}$, Nadine Tinne ${ }^{\mathrm{a}, \mathrm{b}}$, Roland Lachmayer ${ }^{\mathrm{a}, \mathrm{b}, \mathrm{c}}$, Jörg Neumann ${ }^{\mathrm{b}}$, Dietmar Kracht ${ }^{\mathrm{b}}$ \\ ${ }^{a}$ Tailored Light, PhD program, Gottfried Willhelm Leibniz Universität Hannover, 1 Welfengarten, \\ D-30167 Hannover, Germany \\ ${ }^{\mathrm{b}}$ Laser Zentrum Hannover e.V., 8 Hollerithallee, 30419 Hannover, Germany \\ 'Institut für Gerätebau und Produktentwicklung (IPeG), Gottfried Wilhelm Leibniz Universität \\ Hannover, 1 Welfengarten, D-30167 Hannover, Germany
}

\begin{abstract}
A new family of lighting products is developed as laser diodes replace LEDs in the remote phosphor configuration. The resulting lighting systems, also known as laser-excited remote phosphor systems, exhibit advanced characteristics compared to LEDs, such as significantly higher luminance and smaller étendue. However, the bottleneck in their performance is often considered to be the conversion process within the phosphor layer. The high-intensity exciting laser beam in combination with the low thermal conductivity of ceramic phosphor materials leads to thermal quenching, a phenomenon in which the emission efficiency decreases as the temperature rises. In order to investigate the thermal limitations and derive the optimization parameters for these systems, the simulation strategy proposed here effectively takes into account the interplay between the thermal and optical effects. The time-dependent heat equation is solved based on the system's energy balance equation, while the optical effects are modeled within the geometrical optics regime using a ray tracing algorithm. The coupling is achieved considering the temperature-dependent quantum yield (or efficiency) for the phosphor material. For simulation purposes the phosphor material can be considered as a bulk diffuser; the bulk scattering properties are introduced: the absorption and scattering coefficients as well as the scattering (or phase) function. The two-term Henyey-Greenstein function is adopted as scattering function here, since it combines computational efficiency and accuracy. To conclude, an opto-thermal simulation scheme is required for the optimization of a phosphor-converted lighting source. Efficient device design can contribute to the advancement of green lighting technology, a step towards meeting the environmental challenges of our age.
\end{abstract}

Keywords: lighting, laser, remote phosphor systems, modeling, opto-thermal coupling, optimization

\section{INTRODUCTION}

Solid-state lighting technology has revolutionized the way artificial light is produced. Although LEDs show significant advantages compared to conventional light sources, the drop in efficiency as the drive power is increased, a phenomenon known as efficiency droop, presents a fundamental challenge in achieving higher luminance. A solution to this intrinsic limitation of LEDs is the use of laser diodes (LDs) in the remote phosphor process, a configuration ideal for such substitution as the phosphor layer and its exciting optical source are physically separated. Despite the lower electrical to optical power-conversion efficiency of LDs, currently being around $30 \%$ as opposed to that of $70 \%$ for LEDs, their respective power-conversion efficiencies peak for different input power densities. Specifically, while the powerconversion efficiency of LDs is near-zero below the lasing threshold, which is approximately at $4 \mathrm{~kW} / \mathrm{cm}^{2}$, it rises quickly and exceeds that of an LED that peaks at a relatively low input power density (around $\left.3 \mathrm{~W} / \mathrm{cm}^{2}\right)^{1}$. In other words, LDs used as optical pump sources in remote phosphor systems, even when considering their current performance, are a viable alternative that can compete with LEDs in some lighting tasks, especially in applications that require high luminance and étendue limited light sources.

Laser-excited remote phosphor systems have been attracting increasing attention in view of their advanced features over

*e.chatzizyrli@1zh.de; phone +49 511 2788-287; fax +49 511 2788-100; www.lzh.de

Illumination Optics V, edited by Tina E. Kidger, Stuart David, Proc. of SPIE Vol. 10693,

$1069300 \cdot$ C 2018 SPIE · CCC code: $0277-786$ X/18/\$18 · doi: 10.1117/12.2312644 
more traditional lighting solutions. Their significantly higher luminance exceeds even the brightest LEDs by a factor of 2 to $5^{2}$. The much smaller étendue of these light sources maximizes the radiant flux that can be coupled through an optical system, whereas their small light-emitting surface and divergence can also offer great possibilities in creating sharp and exact light distributions. Furthermore, the final lighting device can be downsized and lighter, while the downscaling of the optics used can lead to a cutback of the required resources as well as installation space. In addition, apart from effectively enabling heat dissipation and cooling of the system, the remote phosphor configuration increases design freedom by physically decoupling the optical pump source from the conversion phosphor material. In some set-ups the laser diode(s) can be feet apart from the phosphor layer, whereas an optical fiber may be used for transmitting the laser light.

Although these characteristics have been utilized at some extent in commercial applications, e.g. automotive lighting as well imaging and display applications, laser-based lighting systems have yet to reach their full potential. Apart from the aforementioned low laser diode efficiency issue, the most critical point hindering the output performance of these systems is the thermal stability of phosphors. The high-intensity exciting laser beam combined with the limited thermal conductivity of ceramic phosphor materials gives rise to thermal quenching, the phenomenon in which the emission efficiency decreases with rising temperature. This effect introduces limitations to the lighting devices as the temperature of the phosphor layer cannot exceed a certain threshold, the thermal quenching temperature above which luminescence is quenched. Conversely, the optical system operating on this thermal limit shows optimal efficiency. As evident, in order to investigate the thermal limitations and derive the optimization parameters for these systems, a simulation strategy needs to be devised that effectively takes into account the interplay between the thermal and optical effects. This correlation can be outlined here as follows: the optical heat losses associated with Stokes shift and the non-radiative transitions of luminescent and non-luminescent particles cause a rise in temperature within the phosphor. This in turn results in a decrease in the phosphor's quantum yield until the system reaches its steady-state provided suitable thermal management mechanisms are employed. A simulation model that couples these effects is proposed. While this study focuses on the algorithms implemented, a more in-depth discussion of the modeling aspects of optical and thermal phenomena in laser-excited remote phosphor systems can be found in our previous work ${ }^{3}$. Here, the time-dependent heat equation is solved based on the system's energy balance equation, while the optical effects are modeled within the geometrical optics regime using a ray tracing algorithm. The coupling is achieved considering the temperature-dependent quantum yield (or efficiency) for the phosphor material, more specifically YAG:Ce that is predominantly used in lighting applications. The temperature-dependent quantum yield is derived considering the radiative and non-radiative lifetimes assuming that the former is temperature independent, while the latter satisfies the Arrhenius equation, where the fitted parameters are obtained from experimental data found in literature. For simulation purposes the phosphor material can be considered as a bulk diffuser. Although in principle the scattering problem cannot be addressed by geometrical optics, using wave optics principles the bulk scattering properties are introduced, namely the absorption and scattering coefficients as well as the scattering (or phase) function. The two-term Henyey-Greenstein function is adopted as scattering function here, as it combines computational efficiency and accuracy. Its free parameters are fitted from experimental data that reflect the actual scattering behavior of phosphor particles.

\section{METHODOLOGY}

The primary tool employed in order to study, design and optimize lighting systems is ray tracing software. In recent years, the need to incorporate phosphor-converted light sources in optical system design has led to the development of simulation tools within commercial software that consider the effects of photoluminescence, namely light emission, typically in longer wavelengths, due to photon absorption ${ }^{4,5,6}$. Although steady-state optical analysis is a powerful simulation technique, the disregard of the phosphor's optical properties and temperature interdependence render these software applications of limited use for the optimization process of phosphor-based lighting devices. Approaches in which additional tools are utilized for modeling the thermal effects often have the drawback of leading to un-coupled opto-thermal simulations. Furthermore, other considerations such as a certain rigidity of choice when it comes to the implementation of different scattering profiles and geometry meshing must also be taken into account.

In the biomedical optics community there are several open source Monte Carlo ray tracing software for multi-layered ${ }^{7,8}$ and voxel-based media ${ }^{9}$ as well as mesh-based Monte Carlo solvers ${ }^{10,11}$. Here, a modified version of the Monte Carlo for multi-layered media (MCML) ${ }^{7}$ code is used to include the photoluminescence phenomenon. Photon mitigation and ray propagation can be used interchangeably as phase and polarization effects are ignored; due to multiple scattering these 
are quickly randomized and do not affect the energy transport. The main difference between our model and MCML is the way random events are defined. Instead of considering scattering and absorption at each propagation step, the incident ray may either be elastically scattered or down-converted based on the quantum efficiency value. In case the ray is scattered, only its direction is changed; the new deflection angle is sampled by the two-term Henyey-Greenstein phase function, while absorption is disregarded. If the ray is down-converted, a new wavelength is assigned based on the phosphor's emission spectrum. Furthermore, the ray is scattered homogeneously, whereas absorption is considered according to Equation (1) that accounts for the non-radiative transitions and Stokes shift losses.

$$
\Phi_{\mathrm{abs}}=\left(1-\frac{\lambda_{\mathrm{inc}}}{\lambda_{\mathrm{em}}} \cdot \mathrm{QY}\right) \Phi_{\mathrm{inc}}
$$

where $\Phi_{\mathrm{inc}}$ and $\Phi_{\mathrm{abs}}$ is the incident and absorbed optical flux respectively and QY is the quantum yield (or efficiency) of the photoluminescence process. At last, $\lambda_{\mathrm{inc}}$ and $\lambda_{\mathrm{em}}$ are the wavelength values of the incident and emitted ray; their rate defines the Stokes shift efficiency.

Another reason for the use of MCML as a starting point of the model development is the phosphor's structure. Although laser-excited remote phosphor systems come in two basic configurations: the reflective and transmissive ${ }^{2}$, in both set-ups the phosphor plate as a whole can be considered a layered medium. The phosphor material is bonded to an opaque or a transparent substrate that acts as a heat sink with the additional use of a reflector in the first case of the reflective configuration, see Figure 1. A distinct layer for the bonding material can also be defined as its lower thermal conductivity could hinder the system's cooling mechanisms. As the different layers of the phosphor plate are homogeneous, a mesh-based Monte Carlo solver is deemed unnecessary. Nevertheless, instead of the cylindrically symmetric geometries of the initial MCML program, a tetrahedral mesh is considered as the grid system for depositing the absorbed optical flux and storing the spatially resolved material properties. There are two issues here. First, the material properties at the down-conversion event must be determined based on their values at the nodes of the element that contains its position. Second, the absorbed optical flux at the random position of this down-conversion event must be stored at the nodes of the respective element. Barycentric coordinates are employed to enable these processes ${ }^{12}$. In conclusion, the use of tetrahedral meshes enables the discretization of complex geometries as well as the coupling to the thermal model, where the deposited optical flux is used as volumetric heat source. Thermal analysis is performed by solving the transient heat equation with the use of the finite element method (FEM).

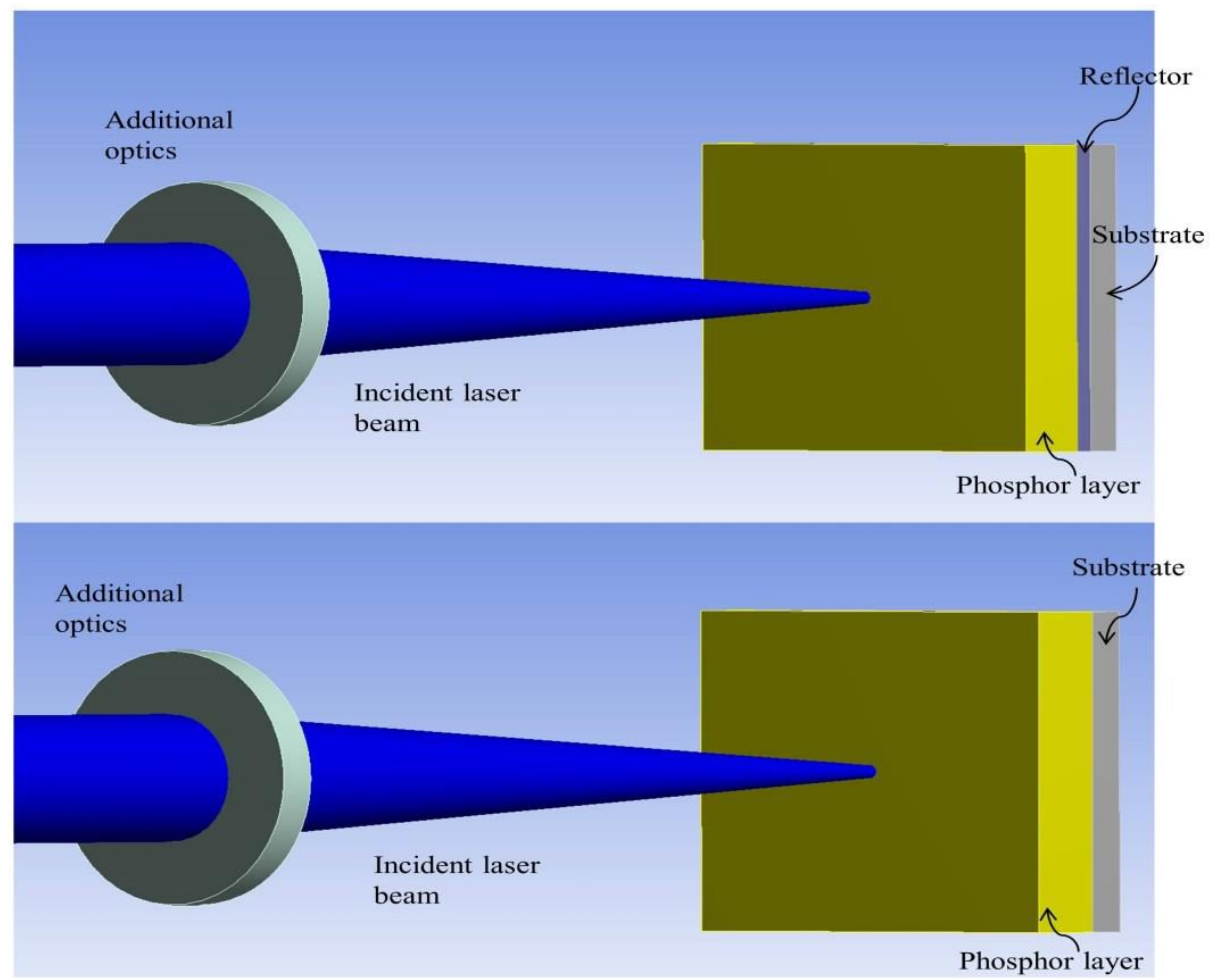

Figure 1. Phosphor plate in reflective (up) and transmissive (bottom) configuration. 
In both reflective and transmissive configurations of the laser-excited remote phosphor systems, a blue emitting laser diode beam (or an array of them) is focused on the phosphor layer. As a result, a focused Gaussian beam is utilized as the model's optical input, whereas its divergence angle, see Figure 1, can be determined by the preceding optical system. As laser light is nearly monochromatic the wavelength dependence of the mean free path length can be neglected.

One of the necessary changes to the MCML code for incorporating the photoluminescence phenomenon is the wavelength assignment to each ray or photon packet. Photoluminescence in this sense is phenomenological: the light conversion process itself is not modeled; rather rays are probabilistically absorbed, down-converted and scattered based on a set of user-provided spectra. Although the absorption and emission spectra of the phosphor material are temperature dependent, these slight shifts can be considered negligible for temperatures below the thermal quenching temperature. Therefore in order to assign a wavelength to each ray, wavelength values at some specified temperature are randomly sampled according to an appropriate probability density function, namely the laser diode and phosphor emission spectrum for the incident and down-converted light respectively, see Figure 2. A common approach to this problem is the discretization of the spectra; each wavelength is emitted based on a certain weight. There are many efficient algorithms for sampling from a discrete probability distribution, e.g. the alias method, that are included in scientific programming libraries.

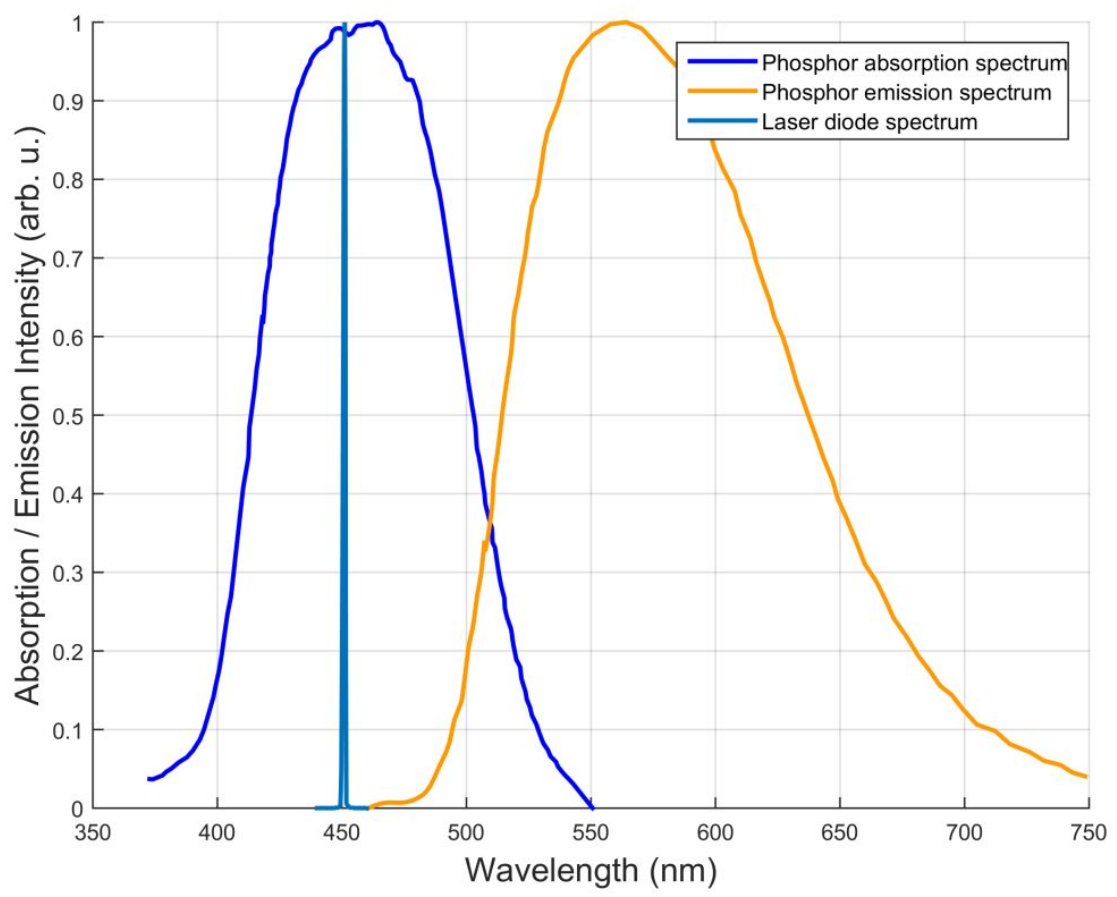

Figure 2. YAG:Ce absorption and emission spectra in conjunction with a blue laser diode emission spectrum that is typically used in lighting applications.

There are several methods for obtaining the bulk scattering properties, namely the absorption $\left(\mu_{\alpha}\right)$ and scattering $\left(\mu_{\mathrm{s}}\right)$ coefficients as well as the phase function $(\mathrm{S}(\theta))$, of the material under study. If the physical properties are known, such as the complex refractive index, particle size distribution, particle shape, concentration and etc., which is hardly the case for commercial phosphors, the bulk scattering properties can be calculated analytically using the Lorenz-Mie theory. Typically, these properties are derived experimentally by measurable quantities such as transmittance, reflectance, regular transmission and spatially resolved reflectance. Iterative procedures such as the inverse Monte Carlo method (IMC) and the inverse adding doubling (IAD) method can be employed that try to find an agreement between simulated and experimental quantities by adjusting the bulk scattering properties for the simulation until a match is reached. An assumption for the scattering function is made; a predefined scattering function is used with one or more free parameter for this iterative procedure. Leyre et al. applied a modified IAD algorithm to samples of YAG:Ce with varying 
concentration to determine their bulk scattering parameters ${ }^{13}$. The comparative study between three predefined scattering functions, those are the Henyey-Greenstein (HG), the two-term Henyey-Greenstein (TTHG) and the Gegenbauer kernel (GK) function, showed that the TTHG scattering algorithm sufficiently models the scattering behavior of phosphor particles. Back scattering can also be considered, something that the one term Henyey-Greenstein function fails to do adequately. Furthermore, it is found that its free parameters $\left(\alpha, g_{1}\right.$ and $\left.g_{2}\right)$ are independent of the YAG:Ce concentration in the sample. The absorption and scattering coefficients on the other hand exhibit a linear correlation with regards to concentration as expected. As a result the two-term Henyey-Greenstein phase function is adopted here, see Equation (2).

$$
\mathrm{S}_{\mathrm{TTHG}}(\mu)=\alpha \frac{1-\mathrm{g}_{1}^{2}}{4 \pi\left(1+\mathrm{g}_{1}^{2}-2 \mathrm{~g}_{1} \mu\right)^{3 / 2}}+(1-\alpha) \frac{1-\mathrm{g}_{2}^{2}}{4 \pi\left(1+\mathrm{g}_{2}^{2}-2 \mathrm{~g}_{2} \mu\right)^{3 / 2}}
$$

where $\mu=\cos \theta$ and $\theta$ the deflection or scattering angle measured with respect to the original ray direction.

The Monte Carlo method is based on the random sampling of propagation variables from well-defined probability distributions. The issue that arises now is how to sample the random variable deflection angle ( $\theta$ or equivalently $\mu$ ) from the TTHG function that serves as its probability density function (PDF). In the available Monte Carlo ray tracing algorithms, where the HG function is adopted, this is easily implemented. The sampling equation, see Equation (3), derived from the 1-to-1 mapping of the cumulative distribution functions (CDFs) of the random variables $\mathrm{R}$ and $\theta$ yields an analytic solution. This technique is also known as the transformation method ${ }^{14}$.

$$
\mathrm{R}=\int_{\mathrm{a}}^{\mathrm{x}} \mathrm{p}(\mathrm{x}) \mathrm{dx}
$$

where $\mathrm{R}$ is a uniformly distributed random number in $[0,1)$ and $\mathrm{p}(\mathrm{x})$ is the probability density function of $\theta$.

When the TTHG phase function is applied in Equation (3), the result is given in Equation (4) ${ }^{15}$. Although the integral part can be solved yielding the respective CDF, this expression cannot be reversed and solved to $\mu$.

$$
\mathrm{R}=\frac{a}{2 g_{1}}\left[\frac{\left(1-g_{1}^{2}\right)}{\left(1+g_{1}^{2}-2 g_{1} \mu\right)^{1 / 2}}-\left(1-g_{1}\right)\right]+\frac{1-a}{2 g_{2}}\left[\frac{\left(1-g_{2}^{2}\right)}{\left(1+g_{2}^{2}-2 g_{2} \mu\right)^{1 / 2}}-\left(1-g_{2}\right)\right]
$$

As a result, Equation (4) is used to form a look-up table that maps the random variable $R \in[0,1)$ to $\mu \in[-1,1]$. Each time a random number is drawn, the look-up table is entered and the appropriate value of $\mu$ is computed using linear interpolation. As the right hand part of Equation (4) is a CDF and therefore sorted, a binary search algorithm is utilized to speed up the process.

\section{OPTO-THERMAL SIMULATION MODEL}

The opto-thermal model presented here follows the basic structure shown in Figure 3. The optical part is implemented by a modified version of the MCML program, while the thermal part is solved using the FEM. As both optical and thermal simulation schemes are computationally expensive, measures must be taken to limit the computation time. Some considerations concerning the termination and optical simulation update criteria can be found in $^{16}$ and are also discussed here: The iterative cycle of opto-themal simulations is terminated when the time derivative of the temperature drops below a certain threshold; small enough to designate that the system has reached its steady-state. The alternative termination criterion is the quantum yield value. When it drops, typically below 0.5 , the system ceases to produce white light. Another issue that needs to be addressed is how often to perform the optical simulation. If the quantum yield does not change much between consecutive runs, namely $\Delta \mathrm{QY}<$ lim', then further thermal simulations are performed. If the quantum yield decreases below lim', then a new optical simulation is launched.

The coupling between optical and thermal effects is achieved by considering the temperature-dependent quantum yield of the phosphor material, see Equation (5). This dependency is described in the following: The phosphor's radiative lifetime $\tau_{\mathrm{r}}$ is considered constant to temperature when compared to the non-radiative lifetime $\tau_{\mathrm{nr}}$. For the calculation of the temperature dependent non-radiative lifetime $\tau_{\mathrm{nr}}$ of YAG:Ce a formula is derived assuming the functional form of $\tau_{\mathrm{nr}}{ }^{-1}$ is the Arrhenius equation ${ }^{17}$, see Equation (6). In Equation (6) $\mathrm{k}_{\mathrm{B}}$ is the Boltzmann constant, whereas the fitted $\mathrm{s}_{\mathrm{o}}$ and $\mathrm{E}_{\mathrm{o}}$ parameters are obtained from experimental data found in literature ${ }^{17}$. 


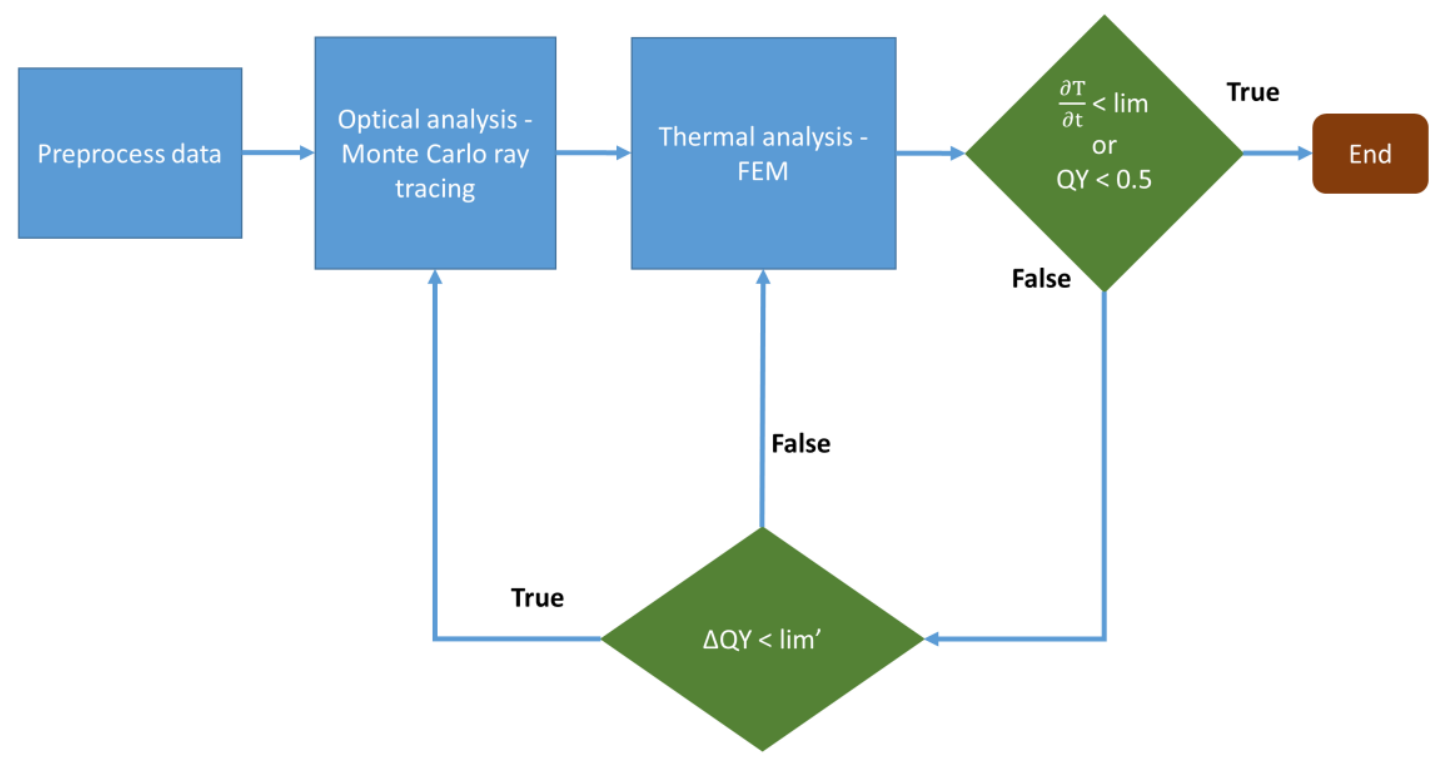

Figure 3. Opto-thermal simulation model flowchart.

$$
\begin{gathered}
\mathrm{QY}(\mathrm{T})=\frac{\tau_{\mathrm{nr}}(\mathrm{T})}{\tau_{n r}(T)+\tau_{r}} \\
\tau_{\mathrm{nr}}(\mathrm{T})=\mathrm{s}_{\mathrm{o}} \exp -\frac{\mathrm{E}_{\mathrm{o}}}{\mathrm{k}_{\mathrm{B}} \mathrm{T}}
\end{gathered}
$$

As already discussed in Section 2, a tetrahedral mesh is adopted for the discretization of the geometry; material properties and optical heat losses are stored at the nodes of each element. Apart from quantum yield, the temperaturedependence of other physical properties, such as the refractive index and thermal conductivity, can also be considered if their relation to temperature is established. In other words, this model offers a way to investigate how a number of parameters affect the system's output performance.

The modified MCML code flowchart is shown in Figure 4. As the MCML code is well documented ${ }^{18}$, we will focus here on the changes introduced to include photoluminescence. Considering a ray that enters the phosphor material, the average distance that the ray travels without hitting a phosphor particle is determined by the mean free path length for photoluminescence. This mean free path, calculated by the absorption $\left(\mu_{\alpha}\right)$ and scattering $\left(\mu_{\mathrm{s}}\right)$ coefficients, is used to determine the step size (s). There are two distinct random events that can occur: scattering or down-conversion. The decision is made based on the quantum yield value, which is defined as the rate of the emitted to the absorbed photons. Since quantum yield is a rate, the random experiment is easily performed by drawing a random number $\mathrm{R} \epsilon[0,1)$ and comparing it to its value. In case of scattering, where any attenuation of the incident ray is disregarded, only the new propagation direction is calculated. When a down-conversion event occurs, the ray is re-emitted at a longer wavelength that is sampled from the phosphor's emission spectrum. Furthermore, as isotropic scattering is considered for the downconverted light, the new deflection angle is determined by uniform sampling from a sphere. It should be noted that for most phosphors used in lighting applications, the absorption and emission spectra overlap, for the case of YAG:Ce see Figure 2. Part of the down-converted light gets reabsorbed without ever contributing to the output white light. As a result, this phenomenon must also be taken into account for obtaining accurate results. Fortunately, this is easily realized without any additional steps. At the decision point for which random event to occur, it is checked whether the incident ray falls within the phosphor's absorption spectrum. Down-converted rays outside this range continue to propagate unhindered. 


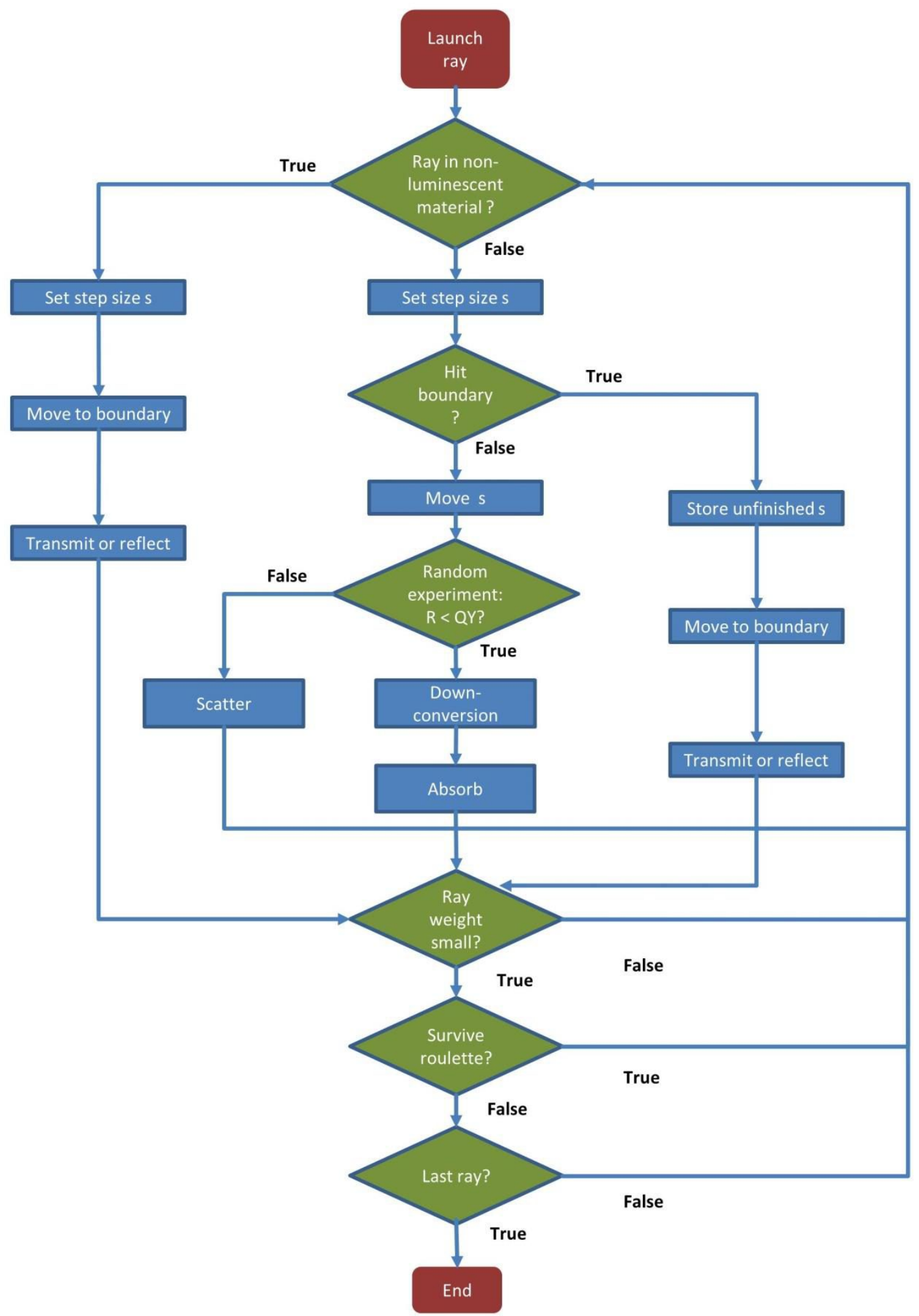

Figure 4. Monte Carlo optical simulation flowchart. 


\section{CONCLUSIONS}

Laser-excited remote phosphor systems are the next stepping stone in solid state lighting, a technology that revolutionized the lighting industry. These systems have the potential to replace LEDs in applications where high luminance and a limited étendue are required. However, their weak point is often considered to be the thermal stability of phosphors; above a certain threshold, the thermal quenching temperature, the light emission efficiency of phosphors drops to practically zero. The mechanism of thermal quenching is outlined as follows: part of the incident light that is neither transmitted through the phosphor material nor down-converted is transformed into heat. Hence, the temperature within the phosphor layer rises and causes a decrease in the quantum efficiency of the conversion process. If appropriate cooling mechanisms are not implemented, the lower quantum efficiency leads to even greater heat losses and consequently to higher temperatures. As can be concluded, the coupling of thermal and optical analysis, which is intrinsically a time-dependent process, is essential for designing and optimizing phosphor-converted light sources.

The opto-thermal simulation strategy proposed here uses a modified version of the MCML program to model the optical effects, while the thermal analysis is performed using the finite element method. Different aspects of the modeling process are discussed with focus on the optical part. As the development of ecologically friendly lighting systems featuring low power consumption and high luminous efficiency becomes imperative given the environmental challenges of our age, a simulation tool that enables efficient device design can be a step towards that aim.

\section{ACKNOWLEDGEMENTS}

The PhD program Tailored Light is funded by the Lower Saxony Ministry for Science and Culture (MWK), Germany.

\section{REFERENCES}

[1] Wierer, J. J., Tsao, J. Y. and Sizov, D. S., "Comparison between blue lasers and light-emitting diodes for future solid-state lighting," Laser \& Photonics Rev. 7(6), 963-993 (2013).

[2] Lenef, A., Kelso, J., Tchoul, M., Mehl, O., Sorg, J. and Zheng, Y., "Laser-activated remote phosphor conversion with ceramic phosphors," Proc. SPIE 9190, 91900C (2014).

[3] Chatzizyrli, E., Tinne, N., Lachmayer, R., Neumann, J. and Kracht, D., "Modeling of photoluminescence in laser-based lighting systems," Proc. SPIE 10603, 1060318 (2017).

[4] Zollers, M., "Phosphor modeling in LightTools: Ensuring accurate white LED models," White paper, January 2011, <https://www.synopsys.com/content/dam/synopsys/optical-solutions/documents/datasheets/modelingphosphors-in-lighttools.pdf > (20 September 2017).

[5] Gangadhara, S., "Modeling a white light source using the phosphor DLL," Zemax knowledgebase, 7 July 2011, $<$ http://customers.zemax.com/os/resources/learn/knowledgebase/modeling-a-white-light-source-using-thephosphor-d> (21 September 2017).

[6] Gay, S., "Overview of photoluminescence simulation in OpticStudio," Zemax knowledgebase, 5 April 2015, $<$ http://customers.zemax.com/os/resources/learn/knowledgebase/overview-of-photoluminescence-simulation-inoptics> (21 September 2017).

[7] Wang, L. H., Jacques, S. L. and Zheng, L. Q., "MCML - Monte Carlo modeling of photon transport in multilayered tissues," Computer Methods and Programs in Biomedicine 47, 131-146 (1995).

[8] Alerstam, E., Svensson, T. and Andersson-Engels, S., "Parallel computing with graphics processing units for high-speed Monte Carlo simulation of photon migration," J. Biomed. Opt 13(6), 060504 (2008).

[9] Qianqian, F. and Boas, D. A., "Monte Carlo simulation of photon migration in 3D turbid media accelerated by graphics processing units," Opt. Express 17(22), 20178-20190 (2009).

[10] Qianqian, F., "Mesh-based Monte Carlo method using fast ray-tracing in Plücker coordinates," Biomed. Opt. Express 1(1), 165-175 (2010).

[11]Qianqian, F. and Kaeli, D. R., "Accelerating mesh-based Monte Carlo method on modern CPU architectures," Biomed. Opt. Express 3(12), 3223-3230 (2012).

[12] Warren, J., Schaefer, S., Hirani, A. N. and Desbrun, M., "Barycentric coordinates for convex sets," Adv. Comput. Math. 27(3), 319-338 (2007). 
[13] Leyre, S., Durinck, G., Hofkens, J., Deconick, G. and Hanselaer, P., "Experimental determination of the absorption and scattering properties of YAG:Ce phosphor," Proc. of SOLED, DTu4C.4 (2014).

[14] Press, W. H., Teukolsky, S. A., Vetterling, W. T. and Flannery, B. P., [Numerical Recipes in C: The art of scientific computing], Cambridge University Press, 287-290 (1993).

[15] Witt, A. N., "Multiple scattering in reflection nebulae: I. A Monte Carlo approach," Astrophys. J. Supplement Series 35, 1-6 (1977).

[16] Correia, A., Hanselaer, P. and Meuret, Y., “An efficient optothermal simulation framework for optimization of high-luminance white light sources," IEEE Photon. J. 8(4), 1601215 (2016).

[17] Lyu, L. J. and Hamilton, D. S., "Radiative and nonradiative relaxation measurements in $\mathrm{Ce}^{3+}$ doped crystals," Lumin. J. Vol. 48/49, 251-254 (1991).

[18] Wang, L. H. and Jacques, S. L., "Monte Carlo modeling of light transport in multi-layered tissues in Standard C," MCML Manual, January 1998, <https://omlc.org/software/mc/man_mcml.pdf> (20 February 2018). 